\title{
Effect of poloidal asymmetry on the impurity density profile in tokamak plasmas
}

\author{
T. Fülöp and S. Moradi \\ Department of Applied Physics, Nuclear Engineering, Chalmers University of Technology and Euratom-VR \\ Association, Göteborg, Sweden
}

(Received 14 January 2011; accepted 22 February 2011; published online 29 March 2011)

\begin{abstract}
The effect of poloidal asymmetry of impurities on impurity transport driven by electrostatic turbulence in tokamak plasmas is analyzed. It is found that if the density of the impurity ions is poloidally asymmetric then the zero-flux impurity density gradient is significantly reduced and even a sign change in the impurity flux may occur if the asymmetry is sufficiently large. This effect is most effective in low shear plasmas with the impurity density peaking on the inboard side and may be a contributing factor to the observed outward convection of impurities in the presence of radio frequency heating. [doi:10.1063/1.3569841]
\end{abstract}

The presence of impurities in fusion plasmas has a significant effect on fusion performance. Accumulation of impurities in the core would have detrimental effect on fusion reactivity due to increased radiation losses and plasma dilution. On the other hand the presence of impurities at the edge can be beneficial, and seeded impurities are often used to create a radiative belt in order to reduce the heat loads on the walls. Therefore, it is of great value to understand the physics mechanisms governing the impurity transport, and to identify plasma conditions in which impurity accumulation in the core can be avoided.

Experimental observations on several tokamaks show that the impurity distribution over the flux surface is often poloidally asymmetric. ${ }^{1-5}$ The asymmetries are usually most pronounced for heavy impurities. In the plasma core, where the collisionality is low, in-out asymmetries can arise due to toroidal rotation or the presence of radio frequency (RF) heating. In the case of rotating plasmas, the poloidal asymmetry of the impurity ion distribution is due to the centrifugal force, which causes the impurities to accumulate on the outboard side of flux surfaces. ${ }^{1,2}$ In the case of RF heated plasmas, the asymmetry is a result of the increase of the hydrogen-minority density on the outboard side. These particles tend to be trapped on the outside of the torus and the turning points of their orbits drift toward the resonance layer due to the heating. The poloidal asymmetry in the hydrogenminority density gives rise to an electric field that pushes the other ion species to the inboard side. In the case of highlycharged impurities, this effect is amplified by their large charge $Z$ (Ref. 3). In the tokamak edge, where the plasma is sufficiently collisional, steep radial pressure or temperature gradients give rise to an in-out asymmetry. ${ }^{6}$ Neoclassical theory also predicts an up-down asymmetry, which is caused by the ion-impurity friction. ${ }^{7}$

Recent experiments have shown that auxiliary heating can influence the impurity convective flux. For example in JET, it has been observed that with ion cyclotron resonance heating (ICRH) the accumulation of high- $Z$ impurities can be avoided. ${ }^{8,9}$ The physical mechanism by which the change of the direction of the impurity convective velocity occurs has not yet been clearly identified, in spite of the various efforts that have been made. ${ }^{9-11}$ In this paper we suggest a mechanism that can give rise to a reduction of the impurity peaking, or even a sign change in the impurity convective flux, based on the asymmetry of the impurity density on the flux surface (which could be due to the presence of ICRH or other reasons). Since impurity transport is usually dominated by drift-wave turbulence, in this work we focus on the effect of the impurity poloidal asymmetry on impurity transport driven by microinstabilities. We assume that the neoclassical processes that cause the asymmetry are not affected significantly by the fact that the cross-field transport is dominated by fluctuations and that the equilibrium part of the electric field is not large enough to cause poloidal asymmetries in the main ion and electron densities. For simplicity we consider only the collisionless, electrostatic case and neglect the effect of rotation.

The quasilinear particle flux for impurities is given by $\Gamma_{z}=-k_{\theta} \Im\left(\hat{n}_{z} \phi^{*}\right) / B$, where $\mathfrak{I}$ denotes imaginary part, $k_{\theta}$ is the poloidal wave-number, $\hat{n}_{z}$ is the perturbed impurity density, $\phi$ is the perturbed electrostatic potential and $\phi^{*}$ denotes its complex conjugate. The perturbed impurity density response in an axisymmetric, large aspect ratio torus with circular magnetic surfaces can be obtained from the linearized gyrokinetic (GK) equation, which in the collisionless case can be written as

$$
\frac{v_{\|}}{q R} \frac{\partial g_{a}}{\partial \theta}-i\left(\omega-\omega_{D a}\right) g_{a}=-i \frac{e_{a} f_{a 0}}{T_{a}}\left(\omega-\omega_{* a}^{T}\right) \phi J_{0}\left(z_{a}\right),
$$

where $g_{a}$ is the nonadiabatic part of the perturbed distribution function, $\theta$ is the extended poloidal angle, $f_{a 0}$ $=n_{a} /\left(\sqrt{\pi} v_{T a}\right)^{3} \exp \left(-x_{a}^{2}\right)$ is the equilibrium Maxwellian distribution function, and $x_{a}=v / v_{T a}$ is the velocity normalized to the thermal speed $v_{T a}=\left(2 T_{a} / m_{a}\right)^{1 / 2}$. The parameters $n_{a}, T_{a}, m_{a}$, and $e_{a}$ are the density, temperature, mass, and charge of species $a, \omega_{* a}=-k_{\theta} T_{a} / e_{a} B L_{n a}$ is the diamagnetic frequency, with $B$ the equilibrium magnetic field, $\omega_{* a}^{T}$ $=\omega_{* a}\left\{1+\left[x_{a}^{2}-(3 / 2)\right] \eta_{a}\right\}$ and $\eta_{a}=L_{n a} / L_{T a}$, where $L_{n a}$ $=-\left[\partial\left(\ln n_{a}\right) / \partial r\right]^{-1}$ and $L_{T a}=-\left[\partial\left(\ln T_{a}\right) / \partial r\right]^{-1}$, are the density and temperature scale lengths. Finally $\omega_{D a}=-k_{\theta}\left(v_{\perp}^{2} / 2+v_{\|}^{2}\right)$ $\times(\cos \theta+s \theta \sin \theta) / \omega_{c a} R$ is the magnetic drift frequency, where $\omega_{c a}=e_{a} B / m_{a}$ is the cyclotron frequency, $\perp_{\|}$and are 
with respect to the equilibrium field, $q$ is the safety factor, $s=(r / q)(d q / d r)$ is the magnetic shear, $r$ and $R$ are the minor and major radii, $J_{0}$ is the Bessel function of the first kind and $z_{a}=k_{\perp} v_{\perp} / \omega_{c a}$. If we neglect parallel compressibility by assuming $k_{\|} v_{T z} \ll \omega$, the gyrokinetic equation can be solved to find

$$
\hat{n}_{z}=\frac{Z e \phi n_{z}}{T_{z}}\left(-1+\int d^{3} x_{z} \frac{\omega-\omega_{* z}^{T}}{\omega-x_{z}^{2} \hat{\omega}_{D z}} \frac{e^{-x_{z}^{2}}}{\pi^{3 / 2}} J_{0}^{2}\left(z_{z}\right)\right),
$$

where $\hat{\omega}_{D z}=\omega_{D z 0} \mathcal{D}(\theta), \quad \omega_{D z 0}=-2 k_{\theta} v_{T z}^{2} / 3 \omega_{c z} R, \quad \mathcal{D}(\theta)=\cos \theta$ $+s \theta \sin \theta$, and we have used the constant energy resonance (CER) approximation $\left[v_{\perp}^{2}+2 v_{\|}^{2} \rightarrow 4\left(v_{\perp}^{2}+v_{\|}\right)^{2} / 3\right]$. Solving the velocity space integrals $\int d^{3} x_{z}(\ldots)$ in Eq. (2) we obtain

$$
\begin{aligned}
\hat{n}_{z}= & \frac{n_{z} Z e \phi}{T_{z}}\left\{-\widetilde{\omega}_{* z}+\left(\frac{3 \widetilde{\omega}_{D z}}{2}-b_{z}\right)\right. \\
& \left.\times\left[\hat{\omega}_{\eta^{* z}}-\frac{5}{2}\left(\eta_{z} \widetilde{\omega}_{* z}-\widetilde{\omega}_{D z} \hat{\omega}_{\eta^{*} z}\right) \mathcal{F}_{7 / 2}^{1}\left(\widetilde{\omega}_{D z}\right)\right]\right\} .
\end{aligned}
$$

Here $\quad b_{a}=b_{a 0}\left(1+s^{2} \theta^{2}\right) \equiv b_{a 0} \mathcal{B}(\theta), \quad b_{a 0}=\left(k_{\theta} \rho_{s a}\right)^{2}, \quad$ and $\quad \rho_{s a}$ $=v_{T a} / \sqrt{2} \omega_{c a}$. The tilde denotes normalization to $\omega$, so that $\widetilde{\omega}_{* a}=\omega_{* a} / \omega, \widetilde{\omega}_{D z}=\hat{\omega}_{D z} / \omega$, and $\hat{\omega}_{\eta_{* a}}=1-\left(1-3 \eta_{a} / 2\right) \widetilde{\omega}_{* a}$. The effect of drift resonances is incorporated in $\mathcal{F}_{b}^{a}(z)$, where $\mathcal{F}_{b}^{a}(z)={ }_{2} F_{0}(a, b ; ; z)$, and ${ }_{2} F_{0}$ denotes the generalized hypergeometric function. An expression similar to Eq. (3) was derived in Ref. 12 but in that case the magnetic drift resonances were replaced with their weighted-averages over an assumed form of the electrostatic potential. Here, we keep the $\theta$-dependence of the magnetic drift frequencies and no ansatz for the perturbed electrostatic potential is used.

The zero-flux impurity density gradient can be obtained from $\Gamma_{z}=0$ with the perturbed impurity density taken from Eq. (3). If the impurity density varies over the flux surface, in general we can write $n_{z}=n_{z 0} \mathcal{P}(\theta)$, where $\mathcal{P}(\theta)=1$ if the impurities are evenly distributed over the flux surface. A poloidal asymmetry can be modeled by $\mathcal{P}^{n}(\theta)=\left\{\cos ^{2}[(\theta\right.$ $-\delta) / 2]\}^{n}$, where $\delta$ is the angular position where the impurity density has its maximum. The peakedness of the asymmetry grows with $n$.

For high- $Z$ impurities, the hypergeometric function can be replaced by its asymptotic limit for small arguments, $\mathcal{F}_{7 / 2}^{1}\left(\widetilde{\omega}_{D z}\right)=1$ (since $\left.\widetilde{\omega}_{D z} \propto 1 / Z\right)$. Then $\left\langle\Gamma_{z}\right\rangle=0$ leads to

$$
\begin{aligned}
\Im\left\{\widetilde{\omega}_{* z}\left\langle\mathcal{P}(\theta)|\phi|^{2}\right\rangle\right\}= & \Im\left\{\left\langle\mathcal{P}(\theta)|\phi|^{2}\left(\frac{3 \widetilde{\omega}_{D z}}{2}-b_{z}\right)\right.\right. \\
& \left.\left.\times\left[\hat{\omega}_{\eta^{*} z}-\frac{5}{2}\left(\eta_{z} \widetilde{\omega}_{* z}-\widetilde{\omega}_{D z} \hat{\omega}_{\eta^{*} z}\right)\right]\right\rangle\right\},
\end{aligned}
$$

where $\langle\cdots\rangle=(1 / 2 \pi) \int_{-\pi}^{\pi}(\cdots) d \theta$. Furthermore, since $\widetilde{\omega}_{* z}$ and $\widetilde{\omega}_{D z}$ are $O(1 / Z)$, the expression $\hat{\omega}_{\eta * z}-(5 / 2)\left(\eta_{z} \widetilde{\omega}_{* z}\right.$ $\left.-\widetilde{\omega}_{D z} \hat{\omega}_{\eta * z}\right)=1+O(1 / Z)$. In the limit of moderate or high impurity charge, all terms of order $1 / Z^{2}$ can be neglected, so Eq. (4) simplifies to $\Im\left\{\widetilde{\omega}_{*_{z}}\right\}=\Im\left\{3 \widetilde{\omega}_{D z 0}\langle\mathcal{D}(\theta)\rangle_{\phi} / 2\right\}$, where $\langle\cdots\rangle_{\phi}=\left\langle\cdots \mathcal{P}|\phi|^{2}\right\rangle /\left\langle\mathcal{P}|\phi|^{2}\right\rangle$. Using the definitions of the drift and diamagnetic frequencies we thus obtain

$$
\mathfrak{I}\left\{\frac{R}{L_{n z}^{0}} \frac{1}{\omega}\right\}=\mathfrak{I}\left\{\langle\mathcal{D}(\theta)\rangle_{\phi} \frac{2}{\omega}\right\},
$$

where $R / L_{n z}^{0}$ denotes $R / L_{n z}$ for $\left\langle\Gamma_{z}\right\rangle=0$, and is the steadystate impurity density gradient (also called the impurity peaking factor).

If the impurities are evenly distributed over the flux surface, and we assume a flute electrostatic potential ( $\phi$ independent of $\theta$ ), the zero-flux impurity density gradient becomes $R / L_{n z}^{0}=2 s$. This is in agreement with the value obtained keeping all the terms in the expression for the impurity flux [which for the GA standard case ${ }^{13}$ and $Z=10$ is $R / L_{n z}^{0} \simeq 2$ (Ref. 14)]. We note that an up-down asymmetry would generally not change the sign of the transport. In the case of a flute potential it only increases the transport slightly, since if $\delta= \pm \pi / 2$, then $\langle\mathcal{D}(\theta)\rangle_{\phi}=C s$, where $C \geq 1$. However an in-out asymmetry has a considerable effect. Taking for example $n=1$ gives $R / L_{n z}^{0}=2 s+\cos \delta(1-s / 2)$. Interestingly, the sign of the zero-flux impurity gradient changes when the shear $s=2 \cos \delta /(\cos \delta-4)$. If the impurity density is in-out asymmetric $(\delta=\pi)$, the sign of $R / L_{n z}^{0}$ is negative for low shear, and the larger $n$ is, the larger the shear value for which the sign change occurs.

For high $Z$ the zero-flux impurity density gradient does not have an explicit dependence on $Z$, although this can be somewhat misleading since, in general, the strength of the asymmetry depends on $Z$ (usually it is larger for heavy impurities). The fact that the zero-flux impurity density gradient is nearly independent of $Z$ has been noted before, in both fluid and gyrokinetic simulations of ion-temperature-gradient (ITG) turbulence dominated transport, without taking into account the poloidal impurity asymmetries. ${ }^{10,15}$ Our results agree with that, but we note that the independence from $Z$ would disappear if the asymmetry were a strong function of $Z$.

If the electrostatic potential is ballooning, with a sheardependent imaginary part, as assumed in Refs. 12 and 14,

$\phi(\theta)=\phi_{0}\left(\frac{1+\cos \theta}{2}+i f_{s} \sin ^{2} \theta\right)[H(\theta+\pi)-H(\theta-\pi)]$,

where $H$ is the Heaviside function and $f_{s}$ can be written approximately as $f_{s}=-0.6 s+s^{2}-0.3 s^{3}$, then the in-out asymmetry leads again to a significantly reduced zero-flux impurity gradient for low shear. Figure 1 shows $R / L_{n z}^{0}$ as function of shear, both for the case of a poloidally symmetric impurity density and for the case of in-out $(\delta=\pi)$, up-down ( $\delta$ $=\pi / 2)$ and out-in $(\delta=0)$ asymmetric impurity densities for $n=4$. Interestingly, also the up-down asymmetry leads to a slightly reduced zero-flux impurity gradient for low shear in this case.

Clearly, for any assumed form for the electrostatic potential, the zero-flux impurity density gradient depends strongly on the sign and peakedness of the asymmetry. Typically, if the impurities are accumulated on the inboard side, the zero-flux impurity density gradient is much lower that if they are evenly distributed over the flux surface or if they are accumulated on the outboard side. However, for moderate shear, the zero-flux impurity density gradient is expected to 


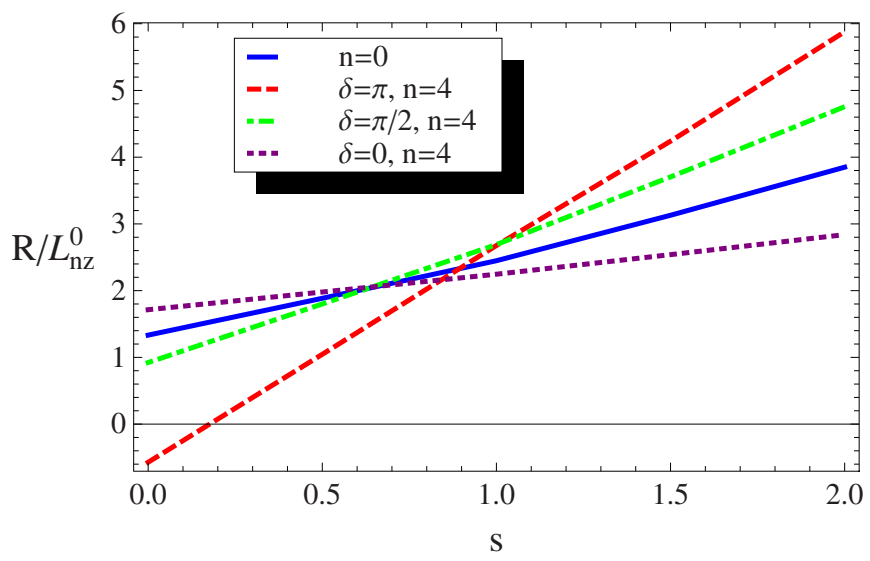

FIG. 1. (Color online) Zero-flux impurity density gradient for high- $Z$ impurities, $R / L_{n z}^{0}=2\langle\mathcal{D}(\theta)\rangle_{\phi}$, as a function of shear.

be positive; partly because if the potential is ballooning on the outboard side it outweighs the effect of impurity accumulation, but also because the sign change tends to occur at low shear.

To calculate the zero-flux impurity density gradient in low shear JET-like core plasmas we have used numerically calculated values for the perturbed potential and eigenvalues for the following parameters: $r / a=0.3, R / a=3, k_{\theta} \rho_{s}=0.3$, $q=1.7, \quad a / L_{n e}=1.3, \quad a / L_{n i}=0.86, \quad a / L_{T e}=0.2, \quad a / L_{T i}=0.3$, $T_{i} / T_{e}=0.85, s=0.22$, and $\rho_{s} / a=0.0034$. These parameters are taken from a typical JET-shot with ICRH heating. ${ }^{9}$ We assume that the impurities are present in trace quantities and do not affect the turbulence characteristics. The absolute value of the potential calculated by $\mathrm{GYRO}^{13}$ is given in Fig. 2, together with the approximate analytical potential from Eq. (6), representative of moderate shear.

The zero-flux impurity gradient calculated from Eq. (5) with the parameters given above is shown in Fig. 3. Figure

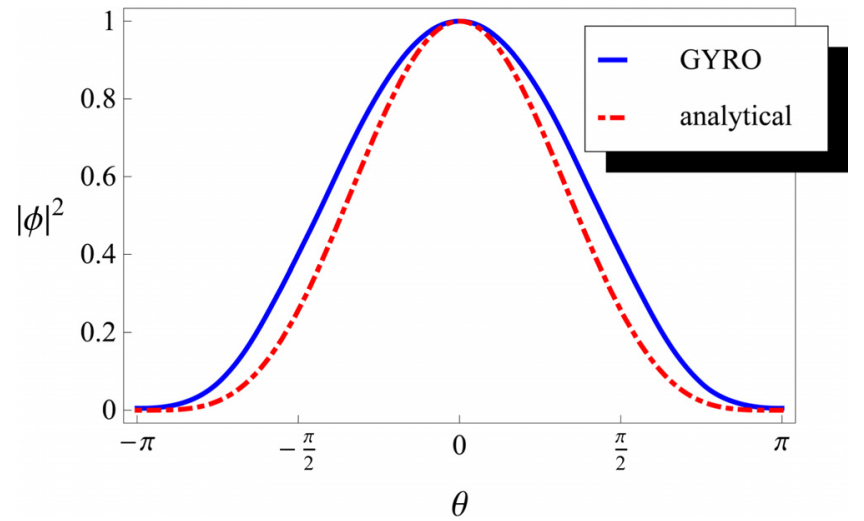

FIG. 2. (Color online) Absolute value of the perturbed electrostatic potential $|\phi|^{2}$ obtained by GYRO for a JET-like case with ICRH, together with the potential given in Eq. (6) which is valid for moderate shear.

3(a) illustrates that for strongly peaked asymmetries if $\delta$ is close to $\pi$ (inboard accumulation), the sign of the zero-flux impurity gradient changes. Figure 3(b) shows that in this particular JET-like case the zero-flux impurity gradient is very sensitive to the peakedness of the asymmetry in the case of in-out asymmetry $(\delta=\pi)$. The sign change occurs around $n \simeq 4$. However, if the impurities are accumulated on the outboard side $(\delta=0), R / L_{n z}^{0}$ grows with $n$, but it is less sensitive to the peakedness of the asymmetry. An up-down asymmetry reduces $R / L_{n z}^{0}$, but not as much as the in-out asymmetry and a sign change is not expected to occur.

Similar calculations for other radial positions, shears, and safety factors give similar results. Our conclusion is that the exact form of the potential or variation in the above given experimental parameters does not change the results described above and the main reduction of the zero-flux impurity density gradient is due to the in-out asymmetry.

For moderate $Z$, the expression for the zero-flux impurity density gradient can be refined to be

$$
\frac{R}{L_{n z}^{0}}=2 \frac{\langle\mathcal{D}\rangle_{\phi}+\frac{2}{1+\hat{\gamma}^{2}} \frac{k_{\theta} \rho_{s}}{Z \tau_{z} \omega_{0}^{n}}\left(\frac{R}{L_{T z}}\langle\mathcal{D}\rangle_{\phi}-\frac{10}{3}\left\langle\mathcal{D}^{2}\right\rangle_{\phi}\right)+\frac{\left(k_{\theta} \rho_{s}\right)^{2}}{2 Z \tau_{z}}\left(\frac{R}{L_{T z}}\langle\mathcal{B}\rangle_{\phi}-\frac{10}{3}\langle\mathcal{B D}\rangle_{\phi}\right)}{1-\frac{4}{1+\hat{\gamma}^{2}} \frac{k_{\theta} \rho_{s}}{Z \tau_{z} \omega_{0}^{n}}\langle\mathcal{D}\rangle_{\phi}-\frac{\left(k_{\theta} \rho_{s}\right)^{2}}{Z \tau_{z}}\langle\mathcal{B}\rangle_{\phi}},
$$

where $\tau_{z}=T_{e} / T_{z}, \hat{\gamma}=\gamma / \omega_{0}$ is the normalized growth rate and $\omega_{0}^{n}$ is $\omega_{0}$ normalized to $c_{s} / R$. Note that we only neglected terms of order $1 / Z^{3}$ in Eq. (4). Although this approximation is acceptable for rather low values of $Z$, Eq. (7) is not reliable for very low $Z$, e.g., for helium. The expression is valid for both trapped electron (TE) and ITG mode driven turbulence, and there is no assumption on the sign or the magnitude of the eigenfrequency or on the magnitude of any other parameters. The largest term in Eq. (7), which usually gives rise to an inward flux in the poloidally symmetric case (positive $R / L_{n z}^{0}$ ), is the one proportional to $\langle\mathcal{D}\rangle_{\phi}$ originating from the curvature drift. ${ }^{16-18}$ Note that $\langle\mathcal{D}\rangle_{\phi}$ is reduced or even negative if the impurities accumulate on the inboard side (see Fig. 1) and this is the main reason for the reduced $R / L_{n z}^{0}$ for high- $Z$ impurities. The rest of the terms are proportional to $1 / Z$ and are small for high- $Z$ impurities. The terms proportional to $R / L_{T z}$ are due to thermodiffusion and their sign depends on the underlying instability. In the poloidally symmetric case they would give rise to an outward flux if the turbulence is ITG-dominated $\left(\omega_{0}^{n}<0\right)$ and an inward flux in the opposite case. However, in the in-out asymmetric case this is also changed, since, as we have shown, a poloidal 

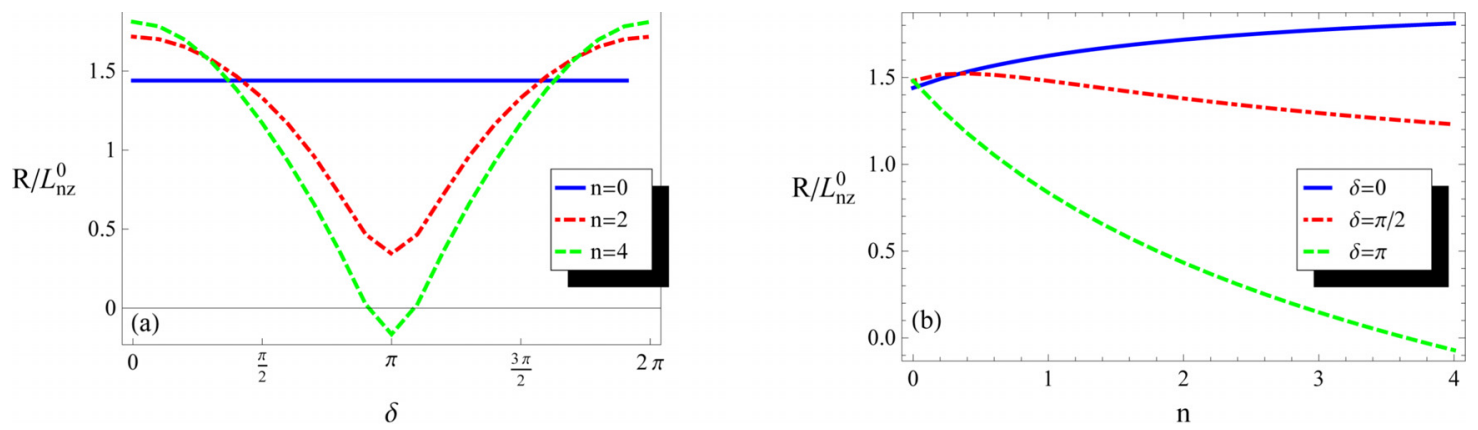

FIG. 3. (Color online) Zero-flux impurity density gradient for the JET-like case: (a) as a function of $\delta$ for three different values of $n$; (b) as a function of $n$ for various values of $\delta$.

accumulation on the inboard side changes the sign of $\langle\mathcal{D}\rangle_{\phi}$. As $\langle\mathcal{D}\rangle_{\phi}$ multiplies $R / L_{T z}$, this sign change leads to an inward flux due to thermodiffusion in the ITG-dominated case. The terms proportional to $\left(k_{\theta} \rho_{s}\right)^{2}$ stem from the finite Larmor radius (FLR) parameter and are usually negligibly small unless $\hat{\gamma}$ is very large. However, that rarely occurs unless $\omega_{0}^{n}$ is very small, in which case the FLR-terms become negligible compared to the terms containing $\omega_{0}^{n}\left(1+\hat{\gamma}^{2}\right)$.

In our orderings, terms corresponding to parallel compressibility are treated as formally small. The contribution due to parallel compressibility to $R / L_{n z}^{0}$ is proportional to $\left(Z \tau_{z} / k_{\theta} \rho_{s}\right)\left(v_{T z} / q R \omega\right)^{2}$ and is usually directed outward for transport driven by TE modes and inward for ITG modes. ${ }^{10}$

The reduction of the zero-flux impurity gradient due to poloidal impurity asymmetries may be a contributing factor to the observed impurity flow reversal in the presence of RF-heating. According to recent observations from JET, ${ }^{9}$ the higher the applied ion cyclotron resonance heating power the lower is the zero-flux impurity density gradient until it eventually changes sign and becomes negative. The reason for this may be that as the ICRH power is raised, the impurities are pushed to the inboard of flux surfaces. The in-out asymmetry can then lead to a change in the impurity flux from inward to outward as demonstrated here. The more asymmetric the distribution becomes, the lower is the zero-flux impurity density gradient. The point is that if the impurities are accumulated on the inboard side (regardless of reason), they will not be sucked into the plasma by the combined effect of the $\theta$-dependence of their density, magnetic drift frequency, and the electrostatic potential. The physical reason for this is that the magnetic drift frequency changes sign at the inboard side, so if the impurities are mainly accumulated there, their averaged flux will change sign. We have shown that the sign change occurs for low shear, so the effect should be more pronounced in the core region. The main mechanism that produces in-out asymmetries in the core is the RF-induced increase of the hydrogen-minority density on the outboard side which gives rise to an electric field that pushes the impurities to the inboard side.

The analysis in this paper applies for a large aspect ratio torus with circular cross section, and the magnitude of the effect may change for realistic equilibra. Also, the effect of rotation, which is one of the mechanisms that causes poloidal asymmetry, has not been taken into account. Fluid and gyrokinetic modeling of rotation induced contributions to the transport fluxes, ${ }^{19,20}$ have shown that in the ITG-dominated case, the centrifugal force leads to higher inward convection for impurities (increasing with impurity mass). This agrees with the predictions of the present work, since the strength of the asymmetry increases with $Z$, and accumulation at the outboard side should lead to an increased inward flux.

The authors gratefully acknowledge helpful conversations with P. Helander, I. Pusztai, S. Newton, and H. Nordman, and would like to thank J. Candy for providing the GYRO code. This work was funded by the European Communities under Association Contract between EURATOM and Vetenskapsrådet.

${ }^{1}$ J. A. Wesson, Nucl. Fusion 37, 577 (1997).

${ }^{2}$ M. Romanelli and M. Ottaviani, Plasma Phys. Controlled Fusion 40, 1767 (1998).

${ }^{3}$ L. C. Ingesson, H. Chen, P. Lender, and M. J. Mantsinen, Plasma Phys. Controlled Fusion 42, 161 (2000).

${ }^{4}$ K. D. Marr, B. Lipschultz, P. J. Catto, R. M. McDermott, M. L. Reinke, and A. N. Simakov, Plasma Phys. Controlled Fusion 52, 055010 (2010).

${ }^{5}$ I. Condrea, E. Haddad, C. Cote, and B. C. Gregory, Plasma Phys. Controlled Fusion 43, 71 (2001).

${ }^{6}$ T. Fülöp and P. Helander, Phys. Plasmas 6, 3066 (1999).

${ }^{7}$ C. T. Hsu and D. J. Sigmar, Plasma Phys. Controlled Fusion 32, 499 (1990).

${ }^{8}$ M. E. Puiatti, M. Valisa, M. Mattioli, T. Bolzonella, A. Bortolon, I. Coffey, R. Dux, M. von Hellermann, P. Monier-Garbet, M. F. F. Nave, J. Ongena, and contributors to the EFDA-JET Work programme, Plasma Phys. Controlled Fusion 45, 2011 (2003).

${ }^{9}$ M. Valisa, C. Angioni, L. Carraro, I. Coffey, I. Predebon, M. E. Puiatti, L. Lauro Taroni, B. Alper, P. Belo, C. Giroud, D. Van Eester, E. Lerche, V. Naulin, T. Tala, M. Tsala, and JET-EFDA contributors, Proceedings of the 36th EPS Conference on Plasma Phys. Vol. 33E, Sofia, 2009.

${ }^{10}$ C. Angioni and A. G. Peeters, Phys. Rev. Lett. 96, 095003 (2006).

${ }^{11}$ H. Nordman, R. Singh, T. Fülöp, L.-G. Eriksson, R. Dumont, J. Anderson, P. Kaw, P. Strand, M. Tokar, and J. Weiland, Phys. Plasmas 15, 042316 (2008).

${ }^{12}$ I. Pusztai, T. Fülöp, J. Candy, and R. J. Hastie, Phys. Plasmas 16, 072305 (2009).

${ }^{13}$ J. Candy and R. E. Waltz, J. Comput. Phys. 186, 545 (2003).

${ }^{14}$ T. Fülöp, S. Braun, and I. Pusztai, Phys. Plasmas 17, 062501 (2010).

${ }^{15}$ T. Fülöp and H. Nordman, Phys. Plasmas 16, 032306 (2009).

${ }^{16}$ C. Estrada-Mila, J. Candy, and R. E. Waltz, Phys. Plasmas 12, 022305 (2005).

${ }^{17}$ X. Garbet, N. Dubuit, E. Asp, Y. Sarazin, C. Bourdelle, P. Ghendrih, and G. T. Hoang, Phys. Plasmas 12, 082511 (2005).

${ }^{18}$ S. Futatani, X. Garbet, S. Benkadda, and D. Dubuit, Phys. Rev. Lett. 104, 015003 (2010).

${ }^{19}$ Y. Camenen, A. G. Peeters, C. Angioni, F. J. Casson, W. A. Hornsby, A. P. Snodin, and D. Strinzi, Phys. Plasmas 16, 012503 (2009).

${ }^{20}$ F. J. Casson, A. G. Peeters, C. Angioni, and Y. Camenen, W. A. Hornsby, A. P. Snodin, and G. Szepesi, Phys. Plasmas 17, 102305 (2010). 